[0212-7199 (2005) 22: 4; pp 194-196] ILES DE MEDICINA INTERNA Copyright (C) 2005 ARAN EDICIONES, S.L

AN. MED. InTERnA (Madrid) Vol. 22, N. ${ }^{\circ} 4$, pp. 194-196, 2005

\title{
Síndrome de Pancoast (tumor de sulcus pulmonar superior): revisión de la literatura
}

\author{
P. KHOSRAVI SHAHI
}

Servicio de Oncología Médica. Hospital General Universitario Gregorio Marañón. Madrid

\author{
PANCOAST'S SYNDROME (SUPERIOR PULMONARY SULCUS \\ TUMOR): REVIEW OF THE LITERATURE
}

\section{RESUMEN}

El síndrome de Pancoast se produce por la presencia de un tumor en el vértice pulmonar con extensión local hacia el plexo braquial inferior, cadena simpática cervical inferior y primeros cuerpos vertebrales y costillas. Su causa principal es el cáncer de pulmón no microcítico, y cursa con dolor de hombro y síndrome de Horner. La mejor técnica diagnóstica es la punción transtorácica por su localización periférica, y la mejor opción terapéutica es la quimiorradioterapia neoadyuvante seguida de cirugía en los casos resecables.

PALABRAS CLAVE: Pancoast. Tumor de sulcus superior. Síndrome de Horner. Cáncer de pulmón no microcítico.

\begin{abstract}
Pancoast's sindrome is produced by an apical lung tumor, with a local extensión to inferior brachial plexus, paravertebral sympathetic chain, vertebral bodies and first, second ans third ribs. Its major cause is the non-small cell lung cancer, and this syndrome may produce shoulder pain and Horner's syndrome. The best diagnostic method is transthoracic needle aspiration, because of its peripheral location. Neoadjuvant chemoradiotherapy followed by complete surgical excision is the preferred approach to these tumors.
\end{abstract}

KEY WORDS: Pancoast. Superior sulcus tumor. Horner's syndrome. Non-small cell lung cancer.

Khosravi Shahi P. Síndrome de Pancoast (tumor de sulcus pulmonar superior): revisión de la literatura. An Med Interna (Madrid) 2005; 22: 194-196.

\section{INTRODUCCIÓN}

El síndrome de Pancoast consiste en el conjunto de signos y síntomas producidos por la afectación de las raíces nerviosas octava cervical y las dos primeras raíces torácicas y/o sistema simpático cervical por la presencia de una extensión local de una lesión tumoral en el sulcus pulmonar superior (1).

\section{ETIOLOGÍA}

El síndrome de Pancoast está producido principalmente por una neoplasia broncopulmonar, generalmente no microcítico (adenocarcinoma y epidermoide), localizado en el sulcus pulmonar superior o vértice pulmonar. Sin embargo, este síndrome puede obedecer a otras muchas causas menos frecuentes como neoplasias torácicas primarias o metastásicas o diversas causas infecciosas, tal como se detalla en la tabla I.
Aunque es raro que este síndrome sea producido por un cáncer de pulmón microcítico, sin embargo hay casos descritos.

\section{MANIFESTACIONES CLÍNICAS}

El síntoma más frecuente de presentación es el dolor de hombro y/o del brazo ipsilateral al tumor, como consecuencia de la extensión local del tumor a la pleura parietal, plexo braquial inferior, cuerpos vertebrales y las tres primeras costillas. El dolor de hombro se puede irradiar hacia la axila y el borde cubital del brazo. En la mayoría de las ocasiones el diagnóstico se retrasa en varios meses, confundiéndose el cuadro con un síndrome del hombro doloroso. Pero, además del dolor de hombro o brazo puede aparecer un síndrome de Horner asociado, por afectación del sistema simpático cervical y del ganglio cervical inferior. Este síndrome incluye ptosis palpebral,

Trabajo aceptado: 15 de febrero de 2005

Correspondencia: Parham Khosravi Shahi. Servicio de Oncología Médica. Hospital General Universitario Gregorio Marañón. C/ Dr. Esquerdo, 46. 28007 Madrid. e-mail: drkhosravi@hotmail.com 
TABLA I

CAUSAS DEL SÍNDROME DE PANCOAST

ETIOLOGÍA DEL SÍNDROME DE PANCOAST

1. Neoplasias:

-Neoplasias broncopulmonares:

—No microcítico (epidermoide, adenocarcinoma)

Microcítico, raras veces

-Neoplasias torácicas primarias:

Mesotelioma, hemangiopericitoma

- Metástasis pulmonares:

-Cáncer de laringe, vesical, tiroideo...

-Neoplasias hematológicas:

-Amiloidosis, linfomas, plasmocitomas, granulomatosislinfomatoidea...

\section{Procesos infecciosos:}

-Bacterianas:

Neumonías por estafilococos y pseudomonas, actinomicosis torácica

-Tuberculosis pulmonar

- Hongos:

Aspergillus, criptococo...

—Quiste hidatídico ca que permite evaluar con gran exactitud la posible afectación vascular de los vasos subclavios y/o braquiocefálicos (5).

La tomografía por emisión de positrones (PET) es muy útil en la estadificación de las neoplasias de pulmón, sobre todo de los no-microcíticos, puesto que permite realizar una mejor valoración de la afectación ganglionar mediastínica y de las metástasis a distancia $(6,7)$.

El diagnóstico definitivo requiere la demostración anatomopatológica del tumor. La citología del esputo presenta una baja rentabilidad diagnóstica en estos casos, puesto que se tratan de lesiones de localización periférica, siendo diagnósticos sólo en un 15-20\% de los casos.

Se suele recurrir a la fibrobroncoscopia que permite valorar la permeabilidad y las características de la mucosa del árbol bronquial, con la posible toma de muestras, mediante el broncoaspirado y/o biopsia/punción transbronquial de las lesiones sospechosas y de las adenopatías. Debido a la localización periférica del tumor en muchas ocasiones no se consigue visualizar el tumor, siendo la rentabilidad de fibrobroncospia de $40-60 \%$ (8).

Por tanto, la mejor técnica para establecer el diagnóstico de este tumor es la punción transtorácica (9).

Otras pruebas complementarias incluyen la realización de una bioquímica completa con perfil hepático y renal, hemograma con reticulocitos y frotis de sangre periférica que puede poner de manifiesto una mieloptisis, en cuyo caso habrá que recurrir a una biopsia/aspirado de médula ósea y la realización de una RM o TC craneal para descartar metástasis cerebrales.

miosis, enoftalmos y en ocasiones anhidrosis facial todos ellos ipsilaterales al tumor.

A medida que avanza el cuadro se puede producir debilidad y atrofia de la musculatura intrínseca de la mano. Otros síntomas que pueden surgir son la disnea, tos, dolor torácico e incluso compresión medular cervical con aparición de paraparesia/paraplejía (1-3).

\section{DIAGNÓSTICO}

Ante la sospecha clínica de síndrome de Pancoast (dolor de hombro y/o síndrome de Horner) se debe realizar una técnica de imagen. La primera prueba a realizar es la radiografía simple de tórax en dos proyecciones (posteroanterior y lateral), que en la mayoría de los casos evidencia la presencia de una masa en el vértice pulmonar. Si ante la alta sospecha clínica la radiografía de tórax es normal, habrá que recurrir a una técnica de imagen de mayor rentabilidad diagnóstica, como es la Tomografía Computerizada (TC) helicoidal de alta resolución.

La TC permite obtener una mejor evaluación de la extensión local del tumor y de la posible presencia de adenopatías mediastínicas, afectación vascular, otros nódulos pulmonares y presencia de metástasis a distancia (hepáticas, suprarrenales, etc.). Por ello, se debe realizar siempre al menos una TC torácica y abdominal superior.

La tesonancia magnética (RM) no aporta una mayor rentabilidad diagnóstica en comparación con la TC, aunque la RM es superior a la TC en la evaluación de la extensión local del tumor (invasión pleural, grasa subpleural, plexo braquial o afectación vascular subclavia) (4).

Más reciente es el empleo de la angioresonancia magnéti-

\section{PRONÓSTICO Y TRATAMIENTO}

El síndrome de Pancoast por definición se presenta en un estadio mínimo de IIB, pues el tumor de Pancoast es un T3 en la clasificación TNM. Los factores de mal pronóstico en el síndrome de Pancoast son:

-Pérdida de peso mayor de 5\%.

-Afectación vertebral.

-Afectación vascular o supraclavicular.

-Estadios IIIA o superior (N2,N3 o M1).

La supervivencia a los 5 años varía en función del estadio del tumor, así para los estadios IIB (T3 N0 M0) es de 45 y de $15 \%$ para estadios superiores (2). La presencia de un T4 (tumor de cualquier tamaño que afecta al mediastino, corazón, grandes vasos, tráquea, carina, esófago o cuerpos vertebrales, derrame pleural maligno o nódulos tumorales pulmonares separados del original en el mismo lóbulo) o la afectación ganglionar mediastínica N2 (adenopatías mediastínicas ipsilaterales o subcarinales) o N3 (adenopatías mediastínicas bilaterales o contralaterales, supraclaviculares o escalénicos) suponen un peor pronóstico (10). Otro factor de mal pronóstico es la presencia de metástasis a distancia M1 (estadio IV), siendo el sistema nervioso central (SNC) uno de los sitios más frecuente de metástasis a distancia.

Por tanto, antes de plantear la posibilidad de cirugía es imprescindible descartar la presencia de metástasis del SNC, mediante la realización de una TC o RM craneal. Asimismo, parece recomendable realizar una PET y/o una mediastinoscopia para descartar la presencia de adenopatías mediastínicas N2/N3 antes de considerar la resección del tumor, puesto que los casos M1, N2/N3 no son candidatos a la cirugía por la pobre supervivencia ( $0 \%$ a los 5 años). 
En los casos resecables (T N0-1 M0) el uso de la quimiorradioterapia combinada neoadyuvante seguido de la resección del tumor parece ser la mejor opción terapéutica, con una menor tasa de recurrencia local y una mejor supervivencia

\section{Bibliografía}

1. Arcasoy SM, Jett JR. Superior pulmonary sulcus tumors and Pancoast's syndrome. New Engl J Med 1997; 337: 1370-6.

2. Komaki R, Roth JA, Walsh GL. Outcome predictors for 143 patients with superior sulcus tumors treated by multidisciplinary approach at the University of Texas MD Anderson Cancer Center. Int J Radiat Oncol Biol Phys 2000; 48: 347-54.

3. Hagan MP, Choi NC, Mathisen DJ. Superior sulcus lung tumors: impact of local control on survival. J Thorac Cardiovasc Surg 1999; 117: 108694.

4. Heelan RT, Demas BE, Caravelli JF. Superior sulcus tumors: CT and MR imaging. Radiology 1989; 170: 637-41.

5. Laissy JP, Soyer P, Sekkal SR. Assesment of vascular involvement with magnetic resonance angiography (MRA) in Pancoast syndrome. Magn Reson Imaging 1995; 13: 523-30.

6. Dwemena BA, Sonnad SS, Anglobaldo JO, Wahl RL. Metastases from non-small cell lung cancer: mediastinal staging in the 1990s. Metaanalysis of PET and CT. Radiology 1999; 213: 530-6.

7. Van Tienteren H, Hoekstra OS, Smit EF. Effectiveness of positron emmision tomography in the preoperative assesment of patients with suspected non-small cell lung cancer: the PLUS multicenter randomized trial. Lancet 2002; 359: 1388-92.

8. Maxfield RA, Aranda CP. The role of fiberoptic bronchoscopy and transbronchial biopsy in the diagnosis of Pancoast's tumor. N Y State J Med 1987; 87: 326-9.
(11-13). Con esta modalidad terapéutica la supervivencia a los 2 años es de $50-70 \%$.

En los casos de afectación de los cuerpos vertebrales puede ser útil recurrir a terapias más agresivas (14-16).

9. Walls WJ, Thornburg JR, Naylor B. Pulmonary needle aspiration biopsia in the diagnosis of Pancoast tumors. Radiology 1974; 111: 99-102.

10. Rush V, Parekh K, Leon L. Factors determining outcome after surgical resection of T3 and T4 lung cancers of the superior sulcus. J Thorac Cardiovasc Surg 2000; 119: 1147-53.

11. Attar S, Krasna MJ, Sonett JR, et al. Superior sulcus (Pancoast) tumor: experience with 105 patients. Ann Thorac Surg 1998; 66: 193-198.

12. Wright CD, Menard MT, Wain JC, et al. Induction chemoradiation compared with induction radiation for lung cancer involving the superior sulcus. Ann Thorac Surg 2002; 73: 1541-1544.

13. Komaki R, Mountain CF, Holbert JM, et al. Superior sulcus tumors: treatment selection and results for 85 patients without metastasis (Mo) at presentation. Int J Radiat Oncol Biol Phys 1990; 19: 31-36.

14. Rusch VW, Giroux DJ, Kraut MJ, et al. Induction chemoradiation and surgical resection for non-small cell lung carcinomas of the superior sulcus: initial results of Southwest Oncology Group Trial 9416 (Intergroup Trial 0160). J Thorac Cardiovasc Surg 2001; 121: 472-483.

15. Barnes JB, Johnson SB, Dahiya RS, Temes RT, Herman TS, Thomas CR Jr. Concomitant weekly cisplatin and thoracic radiotherapy for Pancoast tumors of the lung: pilot experience of the San Antonio Cancer Institute. Am J Clin Oncol 2002; 25: 90-92.

16. Gandhi S, Walsh GL, Komaki R, et al. A multidisciplinary surgical approach to superior sulcus tumors with vertebral invasion. Ann Thorac Surg 1999; 68: 1778-1784. 